\title{
UNA DISCUSIÓN SOBRE NORMAS DERROTABLES
}

\author{
Ricardo Caracciolo \\ Universidad Nacional de Córdoba
}

1. Existe una importante controversia acerca de la naturaleza de las llamadas normas derrotables. Cristina Redondo, en dos interesantes trabajos, sostiene que esa discusión esta permeada por una confusión entre dos tipos de derrotabilidad de normas, a las que voy a denominar aquí «derrotabilidad lógica» (en adelante DL) y «derrotabilidad sustantiva» (en adelante DS), respectivamente [Redondo, 1998; Redondo, 2005]. En ambos casos, lo que en principio está en cuestión es la «derrota» de normas susceptibles de expresarse mediante condicionales generalizados sobre un conjunto de circunstancias -que configuran los antecedentes- y ciertas consecuencias normativas, que constituyen los consecuentes. Es decir, normas generales. ${ }^{1}$

\footnotetext{
${ }^{1}$ CR propone en «Razones y Normas» [Redondo, 2005] una distinción, algo complicada, entre varios tipos de «generalidad» $\mathrm{o}$ «universalidad» de normas: las normas «universales en sentido lógico» tienen que expresarse, desde el punto de vista formal, en enunciados condicionales estrictos. Las que son meramente «generales en sentido semántico» pueden expresarse en condicionales derrotables. A su vez, según CR. la tesis según la cual las normas son «universales en sentido práctico» (lo que significa que su relevancia práctica es uniforme e invariable) implica que se tienen que expresar en condicionales estrictos. Sin embargo, ello no significa, para CR, que las normas «universales en sentido lógico» no sean derrotables en sentido sustantivo. Esta última idea es, precisamente, la que me propongo poner en cuestión. Por lo demás, creo que estas distinciones son, al menos, discutibles por otras razones. Por ejemplo, no es nada clara la categoría de «generales en sentido semántico» porque en la medida en que se refieren -como dice CR- a clases de circunstancias (casos genéricos) tienen que formalizarse usando el cuantificador universal y, en esa medida, son «universales en sentido lógico». La distinción implica, además, que hay normas que carecen de relevancia práctica, lo que a su vez supone que por «norma» se entiende, meramente, un enunciado normativo generalizado. Para evitar confusiones, aclaro que aquí me interesa discutir la noción de una «norma derrotable» válida, es decir, normas que son, o se consideran, relevantes en sentido práctico que son, por otra parte, aquellas cuya existencia cuestiona el particularismo.
} 
Me interesa aquí el caso en el que la consecuencia consiste en el deber de realizar acciones que pertenecen a una clase definida. Parece, sin embargo, que la propia distinción de $\mathrm{CR}$ es producto de un equívoco, que no advierte que la denominada DL es (y sólo puede ser) la contrapartida formal de alguna forma de DS, si es que existe tal cosa como la "derrotabilidad sustantiva" de normas. $\mathrm{O}$, al menos, es lo que intento mostrar.

2. Espero respetar al máximo las ideas de $\mathrm{CR}$ al caracterizar mínimamente de la siguiente manera la distinción que propone:

i) DL: una norma es derrotable lógicamente si, y sólo si, de la constatación de la ocurrencia de un hecho que ejemplifica todas las condiciones explícitamente mencionadas en su contenido, no se infiere que se deba realizar una acción de la clase prevista. Ello significa que la regla del modus ponens no se admite con relación a normas de este tipo. Es más, se puede suponer que, precisamente, el rechazo de esta regla constituye la DL de una norma. Esto es, normas DL son las que se corresponden, de acuerdo a su estructura, a condicionales lógicamente derrotables. A su vez, este rechazo pretende reflejar una intuición básica asociada a la noción de «norma derrotable»: el conjunto de las circunstancias expresamente descriptas en el antecedente de los correspondientes enunciados condicionales, no representan una condición suficiente de la consecuencia normativa, i.e. del deber de actuar. Se supone que, cuando una norma es DL, la cuestión de saber si se debe o no realizar la acción estipulada está sometida a excepciones implícitas, esto es, a condiciones negativas no expresadas en su contenido.

CR agrega a esta caracterización dos observaciones importantes que serán parte del objeto de mi discusión. La primera es que semejantes normas carecen de relevancia práctica uniforme, en el sentido de que la mera ejemplificación de las condiciones explícitas no constituye, por sí sola, razón alguna (ni débil ni fuerte) en favor de la acción que conforma el contenido del deber. En rigor, lo que habría que decir es que ese hecho, esto es, esa ejemplificación, no es una razón para el deber (esto es lo que significa decir 
que no se puede inferir el deber). La idea central indica que ello es así porque la condición de «razón» de esas condiciones es una función del contexto de la acción y no puede determinarse de antemano. De manera que, de acuerdo con ello, una norma DL es inservible en el dominio de la práctica, porque no determina condición alguna de la acción [Redondo, 1998]. ${ }^{2}$ La segunda es que tales normas son irremediablemente inestables. La identificación de una excepción implícita (esto es, la explicitación de otra condición) es equivalente a una alteración de la identidad originaria de una norma DL. Por ello -se afirma- la "derrota" de una norma de este tipo modifica su contenido, de manera que se confunden las etapas de su identificación y de su aplicación [Redondo, 2005].

ii) DS: las características de las normas que CR considera derrotables sustantivamente se pueden mostrar en contraposición a las que son derrotables DL: a) son normas que admiten el modus ponens, esto es, se expresan en condicionales cuyos antecedentes representan condiciones suficientes, en este caso, para inferir deberes de actuar; b) si esto es así, entonces tienen relevancia práctica de manera uniforme, porque la ejemplificación de las condiciones siempre es una razón en favor del deber (o de la acción requerida por el deber); c) la identidad de una norma DS es independiente de su aplicación, porque no está sometida a excepciones implícitas. De ello se sigue que, de acuerdo a $\mathrm{CR}$, la «derrotabilidad lógica» y la «derrotabilidad sustantiva» son categorías excluyentes: una norma no puede ser simultáneamente DL y DS. En verdad, según su propuesta, ambas formas de "derrotabilidad" se asocian a concepciones alternativas de las normas [Redondo, 2005]. Pero no obstante las características a), b) y c), el deber de actuar de cierto modo, que se infiere lógicamente -o la razón para actuar que resulta del deber- puede ser «vencido» o derrotado en una ocasión por una razón $\mathrm{R}$, de acuerdo a un «balance» sustantivo de razones (ello, conforme

${ }^{2}$ Sólo pueden ser útiles -según CR- como meros «recordatorios». Discuto más adelante esta idea. 
a alguna teoría sustantiva de las razones para actuar). Esto es, puede ser que, después de un balance de razones, no exista en modo alguno el deber de actuar de acuerdo con una norma DS. En esto consiste su «derrotabilidad sustantiva». Pero esta «derrota», según CR, tampoco afecta ni la identidad ni (supuestamente) la validez de normas de este tipo [Redondo,2005].

3. Para evaluar esta propuesta, hay necesariamente que distinguir, en primer lugar, dos tipos de deber de realizar una acción p (o dos tipos de razones en favor de una acción p): a) el deber concluyente (o final, o definitivo) de realizar p que, por definición, no es derrotable. El deber concluyente o final es el que resuelve definitivamente la cuestión práctica normativa involucrada en la pregunta de si se debe realizar p o no se debe realizar $\mathrm{p}$ en un contexto particular de acción; b) el deber no concluyente (no-final o no-definitivo) que se puede denominar deber prima facie o protanto de realizar $\mathrm{p}$ (no es relevante aquí la distinción entre estas últimas categorías), el que, por definición, puede ser vencido, esto es, es derrotable. ${ }^{3}$ Un deber no concluyente no resuelve, por ello, la cuestión práctica. No cabe duda que $\mathrm{CR}$, más allá de eventuales diferencias terminológicas, acepta esta distinción. La que es admisible aún cuando se niegue la existencia de deberes concluyentes (esto es, aunque se admita el escepticismo acerca de la posibilidad de resolver cuestiones prácticas).

4. En segundo lugar, cabe observar aquí que, de acuerdo a como se presenta la dicotomía entre estos dos tipos de normas derrotables, no hay espacio para representar la estructura lógica de normas inderrotables, es decir, aquellas de las cuales se pueda inferir un deber concluyente (llamadas

\footnotetext{
${ }^{3}$ Cualquiera sea la caracterización de la idea de un deber prima facie, sobre la que existe una controversia abierta, se admite, en general, que no es un deber concluyente, aunque se pueda transformar en un deber final. Precisamente, lo que está en discusión son las condiciones de esa «transformación». Sobre esta cuestión [Snare, 1974].
} 
en la literatura normas «absolutas»). O, al menos no hay, en la tesis de CR, rasgo estructural alguno que pueda ser utilizado para distinguir normas «absolutas» de normas «derrotables sustantivamente». Más bien, los dos tipos se tienen que expresar -según CR- en enunciados que tienen la forma de condicionales lógicamente inderrotables. En vista de ello alguien podría sugerir que no hay distinción alguna que hacer porque todas las normas «universales» (como denomina a las que se expresan en condicionales estrictos) son derrotables sustantivamente por una razón en contra del deber, al menos en alguna ocasión. $\mathrm{O}$, lo que es lo mismo, ninguna norma podría resolver per se cuestiones prácticas. ${ }^{4} \mathrm{Si}$ fuera así, la distinción entre DL y DS no sólo sería excluyente, sino además exhaustiva de un posible dominio de normas generales.

Pero si no se confunde la cuestión conceptual con la pregunta acerca de si existen o no normas inderrotables, no se advierte por qué la idea de una norma absoluta [Shafer-Landau, 1997] no podría reflejarse en un rasgo formal de esas normas, que vaya más allá de la mera constatación de que, en la práctica, no han sido nunca derrotadas, lo que a lo sumo se trataría de un registro empírico irrelevante para la cuestión normativa, o del truismo según el cual, normas inderrotables son las que no pueden derrotadas. Por el contrario, parece que, en el trabajo de CR, el comportamiento lógico de las normas absolutas, a pesar de su innegable diferencia en punto a la resolución de cuestiones prácticas, tiene que ser exactamente el mismo del que se adjudica a las que son derrotables sustantivamente. Notése que, en cambio, sí se postula esa distinción formal entre los dos tipos de normas derrotables. Creo que aquella identificación es producto del mismo equívoco que conduce -en sentido inverso- a distinguir dos tipos de normas derrotables: una falta de correspondencia entre la forma lógica y la idea material que se intenta

\footnotetext{
${ }^{4}$ No es seguro lo que CR piensa al respecto. Pero obsérvese que la siguiente afirmación: «Las conclusiones que se obtienen por la aplicación individual de un condicional normativo, por ejemplo, mediante modus ponens, nunca pueden llamarse concluyentes» que aparece en [Redondo, 1998, n.15, p.251, el resaltado es mío], implica negar la existencia de normas absolutas.
} 
representar. Para mostrarlo, hay que volver ahora a la cuestión de saber si las categorías DL y DS son, desde ese punto de vista material o sustantivo, realmente excluyentes.

5. Voy a comenzar examinando los rasgos que se adjudica a las normas DL. El primero es el rechazo de la regla del modus ponens. Pero hay que precisar que ese rechazo va dirigido a representar en el cálculo la intuición de que pueden existir normas de las cuales no se infieren deberes concluyentes (seguramente, en virtud de alguna teoría sustantiva del deber o de la racionalidad): [Alchourrón, 1996 (1); Pietrovski, 1993]. O lo que es equivalente, el conjunto de circunstancias descriptas en el antecedente de condicionales derrotables destinados a representar esas normas, no representan condiciones suficientes de deberes concluyentes. Los deberes que aparecen en los consecuentes de esos enunciados sólo pueden ser prima facie. De manera correlativa, sus antecedentes son aptos para representar circunstancias que sólo son razones prima facie en favor de un deber concluyente (o de la acción que constituye su contenido) [Alchourrón, 1996 (2)]. Pero, a la inversa de lo que sugiere CR, la relación que una norma DL establece entre una razón prima facie y un deber concluyente es uniforme e invariable, porque se expresan en enunciados cuantificados universalmente con respecto a las circunstancias de la acción [Alchourrón, 1996 (2)]. No hay nada, entonces, en la noción de condicional normativo derrotable que permita inferir que la relevancia de semejantes razones se restringe a los contextos de acción.

Sin embargo, es verdad que CR no interpreta de esta manera a los condicionales derrotables y vale la pena detenerse en este punto: su objetivo explícito es el diseño de una noción de «norma» compatible con la tesis del particularismo [Redondo,2005]. Es decir, según su propia propuesta, la noción de una generalización que no exprese relaciones «universales legaliformes», sino, más bien, que funcione como un «mero resumen o recordatorio» de la relevancia práctica que una cierta circunstancia puede tener en contextos definidos. $\mathrm{O}$, dicho de otra manera, una generalización que, a partir del 
comportamiento de esa circunstancia en ciertos supuestos particulares de acción, sirva como indicador de esa eventual relevancia en supuestos semejantes. Pero esta idea equivale a conceder a las generalizaciones de este tipo una función epistemológica, como lo indica expresamente el mismo J. Dancy [Dancy, 1999], a quién CR cita para avalarla [Redondo, 2005, n.11]. Para Dancy, nada impide que el particularismo admita enunciados generales de esta clase, que eventualmente registren la relevancia probable de una circunstancia. Ello así porque semejantes enunciados generales sólo pueden resultar de descripciones de esa relevancia y, por lo tanto, a la inversa de lo que supone $\mathrm{CR}$, no hay manera de concebirlos como «normas». Porque, si hay alguna distinción que hacer entre descripción y prescripción, ${ }^{5}$ la función de una generalización normativa tiene que consistir, al menos, en la determinación -y no en la descripción- de la corrección de una clase de acciones o de la relevancia de una clase de circunstancias. ${ }^{6}$ Precisamente, sin embargo, el particularismo extremo, al que adhiere Dancy, rechaza

${ }^{5}$ Esta distinción puede eliminarse postulando, verbigracia, que las normas son genuinas proposiciones, susceptibles de clasificarse en la dicotomía verdad-falsedad. Pero es notorio que CR no adhiere a esta alternativa semántica.

${ }^{6}$ Para evitar confusiones, vale la pena distinguir aquí entre una «generalización normativa» y una «generalización empírica», en punto a cómo se relacionan con los hechos mencionados en sus contenidos. La relevancia práctica (normativa) de una generalización normativa, i.e. una norma (derrotable o inderrotable), no es afectada por un caso de ineficacia o de incumplimiento, esto es, por supuestos en los que, circunstancialmente, fracasa en su función de guiar la acción. Por el contrario, una generalización empírica o, en general, descriptiva, tiene que considerarse falsa, a menos que se trate de una generalización probabilística, si los hechos no se corresponden con su contenido. Con frecuencia, esta distinción no se tiene en cuenta en algunas discusiones del particularismo ético cuando se aducen casos de la práctica moral para poner en cuestión la posibilidad de formular generalizaciones que relacionen propiedades empíricas con propiedades éticas [un ejemplo notable, en Little, 2000]. Pero los contraejemplos de una práctica sólo son relevantes para descartar la «normatividad» general de un enunciado, si se entiende que las reglas morales, que establecen esas relaciones, constituyen algún tipo de generalización acerca de hechos. La invariancia de la relevancia práctica a la que se alude en esta discusión resulta, en todo caso, de una generalización normativa. Por ello, si la norma derrotable en cuestión se considera válida esa relevancia no se altera de acuerdo a las circunstancias de la acción. 
cualquier determinación anticipada de la relevancia normativa (de nuevo, Dancy, 1999). Tampoco puede aceptar, entonces, esa función práctica de las normas generales, ni siquiera de las que se califican como "derrotables".? Por consiguiente, o bien las generalizaciones que se aceptan en el dominio de la práctica son compatibles con esta concepción del particularismo, pero entonces no pueden ser normativas. O son normativas y, en esa medida, incompatibles con el particularismo.

Por lo demás, es cierto que en la medida de esa distinción, los condicionales derrotables normativos -esto es, los que se construyen usando operadores deónticos- no son aptos para expresar descripciones y, en consecuencia, tampoco pueden servir para expresar «recordatorio» alguno. Lo que significa que si se admite esa función de las normas -en contra de ese tipo de particularismo-, y no se las confunde con generalizaciones descriptivas, también se puede aceptar que las razones «prima facie», una condición que las normas derrotables determinan en forma general con relación a clases de circunstancias, son también razones en favor de ciertos comportamientos. No es verdad, entonces, como afirma CR con relación al segundo rasgo que les atribuye, que las normas DL carezcan de relevancia práctica uniforme: ${ }^{8}$ al menos, pueden expresar razones no concluyentes. Es obvio que CR, al aceptar la existencia de deberes no concluyentes tiene que aceptar, en consecuencia, la posibilidad de razones

\footnotetext{
${ }^{7}$ Aunque CR no lo señala, es verdad que la concepción «particularista» de la moral o de la razón práctica no es homogénea. La versión fuerte niega a las normas o reglas cualquier función práctica, incluso la de identificación de propiedades o rasgos del mundo normativamente relevantes. La versión más débil acepta que la relevancia pueda ser fijada por normas «prima facie» o derrotables, pero rechaza que las reglas puedan determinar deberes concluyentes [sobre esta cuestión, Shafer-Landau, 1997, pp. 588 y ss.]. Por lo demás, como se sabe, Dancy rechaza la idea de «deber prima facie».

${ }^{8}$ En la idea de C. Alchourrón (que CR no discute) el aporte de una razón prima facie a la cuestión práctica consiste en que constituye una condición contribuyente (una condición necesaria de una condición suficiente) de un deber final [Alchourrón, 1996 (2)]. Al menos esta alternativa le concede «carácter práctico» a las normas DL, salvo que se postule (lo que CR no hace) que la relevancia práctica de las «razones» se limita a las que son concluyentes.
} 
prima facie, al menos en el sentido descripto. Ello resulta de su propia noción de norma "derrotable sustantivamente".

Finalmente, la tesis de la identidad inestable de las normas DL es algo confusa, aún obviando la cuestión problemática de qué significa «aplicar» una norma DL. ${ }^{9}$ Porque supónganse, v.gr., los siguientes enunciados: E1= "Si B entonces se debe hacer p" y E2= "Si B y -C entonces se debe hacer p"; y se admite que E1 expresa la norma DL N1. Pero si E1 expresa una norma completa (aunque derrotable lógicamente, en donde el deber es prima facie), entonces E2 tiene que expresar otra norma N2 (derrotable o inderrotable). Por lo tanto, en presencia de un supuesto de $\mathrm{C}$, la norma que no es aplicable porque se ejemplifica una condición negativa es, claramente, N2. Sin embargo, ello no afecta ni puede afectar la identidad de N1. Ahora bien, si se responde a esta objeción indicando que E2 se limita a explicitar el contenido implícito de una norma DL y que, por consiguiente, N2 es la única norma que puede ser aplicada o no aplicada, ello equivale a decir que E1 no expresa norma alguna. Pero entonces, no existe la norma N1 asociada con ese enunciado, antes de la operación de explicitación, cuya identidad pueda ser modificada o no modificada en el acto de su aplicación. Como el mismo razonamiento vale con respecto cualquier norma DL, se sigue que -en esta alternativa- las llamadas normas derrotables lógicamente sólo son pseudonormas, que no pueden ser aplicadas ni dejadas de aplicar. Por lo tanto, también la supuesta norma N2 es aplicable o no aplicable sólo si su formulación concluye la identificación de una norma no derrotable lógicamente. Es lo que cabe esperar, si se considera, como ocurre en la discusión de normas derrotables, que las "condiciones implícitas" son condiciones del deber final o concluyente de realizar una cierta acción $p .{ }^{10}$

6. Veamos ahora qué sucede con las normas derrotables en sentido sustantivo. En primer lugar, como los deberes mencionados en el contenido

\footnotetext{
${ }^{9}$ Es problemática porque el modelo estándar de «aplicación» de normas generales supone el uso de la regla del modus ponens. Sin embargo, no voy a tratar aquí esta cuestión.

${ }^{10}$ Discuto con más detalle este argumento en el trabajo «¿Existen las normas generales?», actualmente en elaboración.
} 
de normas semejantes pueden ser «derrotados» por alguna razón en contra de la acción, es obvio que no se trata de deberes concluyentes. O, lo que significa lo mismo, las normas DS no determinan deberes finales. ${ }^{11}$ Precisamente, esto es lo que significa decir que se trata de normas derrotables. Pero, entonces, tampoco aquí, al igual que en el supuesto de normas DL, el conjunto de circunstancias que configuran el antecedente de las normas condicionales DS constituye una condición suficiente de un deber final: su uso no habilita per se la resolución de cuestiones prácticas. El deber que constituye el consecuente de una norma DS sólo puede ser, en consecuencia, un deber prima facie y, correlativamente, si esas circunstancias son "razones" en favor del deber final, sólo pueden ser razones prima facie. Como ello es así, hay una cierta incoherencia en negar carácter práctico a las normas DL y concederlo a las normas DS. En todo caso, es claro que de las normas DS no se pueden inferir lógicamente deberes concluyentes. $\mathrm{O}$, lo que es lo mismo, el uso de la regla del "modus ponens" no es admisible para obtener deberes finales a partir de normas DS. Esta es una consecuencia conceptual de la propia noción de norma derrotable. Por lo tanto, como esa misma incapacidad inferencial es la característica que conduce a eliminar esa regla en las normas DL, la afirmación de CR según la cual el rasgo estructural distintivo es su aceptación en las normas DS, constituye una mera postulación incompatible con la atribución de idénticas consecuencias normativas, en cuanto a la naturaleza del "deber", a ambos tipos de normas derrotables. No hay ninguna razón por la cual el uso del modus ponens tenga que ser excluido sólo en las normas DL. ${ }^{12}$

${ }^{11} \mathrm{CR}$ usa con frecuencia la expresión «consecuencia deóntica» para referirse al consecuente de condicionales destinados a expresar normas "universales". Pero es claro que, de acuerdo con su propia caracterización de la idea de una norma "derrotable sustantivamente", esas consecuencias no pueden ser, en ningún caso, deberes concluyentes o finales.

${ }^{12}$ En la lógica de condicionales derrotables (el sistema AD) propuesto por Carlos Alchourrón, tampoco se admite el modus ponens para inferir deberes prima facie [Alchourrón, 1996 (2)]. CR podría cuestionar esta idea, y sugerir que la regla de inferencia tendría que ser aceptada para obtener deberes no concluyentes. Pero una eventual discusión sobre las reglas de inferencia admisibles en el cálculo de normas derrotables no sirve para mantener la distinción entre normas DL y DS: en ambas categorías el «deber» que constituye el consecuente del correspondiente enunciado condicional -como se ha mostrado- es un deber prima facie. Por lo tanto, en los dos tipos tendrían que valer las mismas reglas de inferencia. 
7. En segundo lugar, CR afirma que no se altera la identidad de las normas DS -en contraposición a las que son DL-, aunque el deber que se infiere de las mismas pueda ser «vencido» en alguna ocasión. Pero el mismo hecho de que ese deber pueda ser derrotado, lo que también es una consecuencia de una característica conceptual de normas semejantes, muestra que la obtención de un deber final o, lo que es lo mismo, la resolución de una cuestión práctica, está condicionada, en relación a cada norma DS, a circunstancias que no se expresan en sus contenidos. Por consiguiente, los deberes concluyentes son dependientes aquí de condiciones «implícitas», en el mismo sentido en el que lo son en el caso de las que son derrotables lógicamente. Esto es, en el sentido según el cual esas condiciones no se describen en el contenido originario de esas normas. Supóngase, entonces, que la presencia de una cierta circunstancia configura una razón $\mathrm{R}$, en relación a una norma $\mathrm{N} 1=$ «Si $\mathrm{C}$ entonces se debe realizar $\mathrm{p} »$, derrotable sustantivamente, que bloquea el paso al deber concluyente de realizar $\mathrm{p}$. Como la constatación del hecho de que $\mathrm{R}$ es una razón en contra de $\mathrm{p}$ puede generalizarse, ${ }^{13}$ sería verdad, entonces, que se debe concluyentemente realizar p sólo si, al menos, la situación se describe como "C y no-R". ${ }^{14}$ La pregunta es, entonces: la condición del deber final descripta como no-R, ¿altera o no

\footnotetext{
${ }^{13}$ No es relevante para el argumento la eventual objeción según la cual, en este caso, el «peso» de R, o su propia condición de «razón», se restringe al contexto (algo que CR no dice en sus trabajos). Ello, porque no hay razón alguna para sostener que las circunstancias que constituyen condiciones «implícitas» se generalizan cuando se trata de normas DL, pero no se generalizan en el supuesto de normas DS. O viceversa. No hay aquí tampoco un criterio de distinción. En todo caso, postular que las normas pueden ser «derrotadas» por razones meramente contextuales podría poner en cuestión la propia idea de «norma general»y, por consiguiente, afectar a ambas categorías de normas derrotables. Efectivamente, CR supone que la idea de norma derrotable lógicamente conduce a -o es compatible con- posiciones particularistas [Redondo, 2005]. Pero no advierte que al mismo resultado, si se lo admite, hay que arribar con respecto a las que son derrotables sustantivamente. Discuto este problema de mayor alcance, en el trabajo «¿Existen las normas generales?», mencionado arriba.

${ }^{14}$ Omito la complicación de que, obviamente, pueden existir otras razones en contra de $\mathrm{p}$, esto es, otras excepciones implícitas.
} 
la identidad de N1? La respuesta tiene que ser negativa -como sostiene CRúnicamente si no-R se considera una condición explícita incorporada en otra norma N2 distinta de N1. Si, por el contrario, se considera que N2 se limita a especificar condiciones implícitas de $\mathrm{N} 1$, en relación al deber final de realizar $\mathrm{p}$, la respuesta tiene que ser positiva. Pero como la misma alternativa se tiene con relación a las normas DL, no existe distinción que hacer, en este aspecto, entre ambas categorías de normas derrotables. ${ }^{15}$

8. Como resulta del análisis precedente, lejos de ser excluyente, la dicotomía entre normas DL y DS es, de hecho, inexistente. Ello es así porque en ambos supuestos el rasgo sustantivo básico consiste en que los antecedentes de los condicionales que se usan para enunciarlas, no configuran condiciones suficientes de deberes finales o concluyentes. Se trata del rasgo sustantivo que el cálculo lógico de condicionales derrotables se propone capturar mediante el rasgo estructural que consiste en eliminar la regla del modus ponens y la ley de refuerzo del antecedente. Esto es, ese cálculo esta destinado, precisamente, a expresar la forma de normas de las que no se infieren deberes de ese tipo, i.e. de las normas que se consideran derrotables sustantivamente. Por lo tanto, aquellas que aquí se denominan normas DL no son otra cosa que la contrapartida formal de la idea sustantiva de derrotabilidad. Hay otro punto de vista desde el cual se puede arribar al mismo resultado: la noción de norma derrotable sustantivamente refleja la

\footnotetext{
${ }^{15}$ Es verdad que CR propone una distinción adicional [Redondo, 2005] entre dos clases de «derrota» de normas: una por la presencia de una condición implícita y la otra por un conflicto con otra norma «vencedora». La primera vendría a corresponder a las normas DL y la otra a las DS. Pero francamente no advierto cuál puede ser la diferencia, si se tiene en cuenta que las condiciones de aplicación de la norma que prevalece funcionan siempre como verdaderas condiciones negativas de la norma derrotada, en relación -hay que insistir en esto- a la obtención del deber final. Así, si se tienen $\mathrm{N} 1=$ «Si C entonces se debe realizar p» y N2= «Si R entonces se debe realizar no-p» y N2 «derrota» a N1, ello implica, de nuevo, que se debe realizar $\mathrm{p}$ únicamente si se da el caso «C y no-R». Por lo tanto, es indiferente para mi argumento cuál pueda ser el origen de una «condición implícita».
} 
idea de David Ross acerca de lo que es un principio prima facie, esto es, un enunciado normativo general que sólo puede determinar deberes prima facie. Pero es exactamente la estructura de la misma idea la que se procura capturar en el cálculo de condicionales derrotables [Alchourrón, 1996 (1)].

No hay, entonces, dos tipos de normas derrotables. En rigor, no existe la categoría de «norma derrotable lógicamente». Precisamente porque -como correctamente afirma CR- la cuestión de saber si existen o no normas derrotables no es un problema de lógica. Es verdad que la postulación de la regla del modus ponens en un caso, pero no en el otro, procura indicar una diferencia. Pero, como se ha mostrado, se trata de una diferencia aparente. La afirmación según la cual de las normas DS se infieren deberes -sin distinción alguna acerca de su naturaleza concluyente o no concluyente- no prueba esa diferencia sino que sugiere, más bien, que se ha incurrido en una confusión acerca de su forma lógica, porque se les adjudica aquellos rasgos estructurales que corresponden a normas absolutas o inderrotables, i.e. aquellas cuyos antecedentes configuran condiciones suficientes de un deber final.

Es cierto también que CR supone que en la controversia universalismo versus particularismo de razones no es preciso considerar si existen o no normas absolutas [Redondo, 2005]. Pero esta tesis no advierte que la alternativa débil del particularismo, según la cual cualquier deber final sólo puede resultar de una evaluación completa, en cada ocasión, de las razones en favor y en contra de una acción [Shafer-Landau, 1997] es totalmente compatible con su idea de norma derrotable, a la inversa de lo que ocurre con su versión extrema. Esa opción particularista sólo se excluye si se admite que existen normas generales inderrotables, porque entonces no sería verdad que para resolver cualquier cuestión práctica se tengan que considerar todas las razones del contexto: la función de una norma de este tipo consiste, precisamente, en conceder relevancia decisiva sólo a algunas de las circunstancias de la acción [Shafer-Landau, 1997]. Otra cuestión distinta -que requiere otra discusión- es 


\section{Ricardo Caracciolo}

la de saber hasta qué punto esa supuesta compatibilidad con el particularismo de las normas derrotables permite conservar -como supone CR [Redondo, 1998]- su condición de normas «genuinas».

\section{Referencias}

Alchourrón, Carlos [Alchourrón, 1996 (1)]: «Detachment and Defeasibility in Deontic Logic», en Studia Logica, 57,1996.

Alchourrón, Carlos [Alchourrón, 1996(2)]: «Para una lógica de las razones Prima Facie», en Análisis Filosófico,16,1996.

Dancy, Jonathan [Dancy, 1999]]: «On the Logical and Moral Adequacy of Particularism», en Theoria, vol.LXV, part.1, 1999.

Little, Margaret [Little, 2000]: “Moral Generalities Revisited”, en Hooker, Brad and Little, Margaret Olivia, Moral Particularism, Clarendon Press, Oxford, 2000.

Redondo, Cristina [Redondo, 1998]: "Reglas "genuinas" y positivismo jurídico", en Analisi e Diritto, 1998.

Redondo, Cristina [Redondo, 2005]: «Razones y Normas», en este volúmen.

Pietroski, Paul [Pietroski, 1993]: «Prima Facie Obligations and Ceteribus Laws in Moral Theory», en Ethics, 103, 1993.

Shafer-Landau, Russ [Shafer-Landau, 1997]: "Moral rules", en Ethics, 107, 1997.

Snare, Frank [Snare, 1974]: "The Definition of Prima Facie Duties", en Philosophical Quarterly, vol.24, issue 96, 1974. 